Classification

Physics Abstracts

$61.46-68.48-68.55$

\title{
Recent Developments of the RBS Technique for the Analysis of Semiconductor Nanostructures
}

\author{
Marina Berti(*), Antonio Vittorio Drigo and Giacomo Torzo \\ Dipartimento di Fisica dell'Università di Padova, Unità INFM, via Marzolo 8, 35131 Padova, Italy
}

(Received March 1; accepted June 30, 1995)

\begin{abstract}
It is well-known that in the case of high lattice mismatch the growth of semiconductor structures proceeds via island formation. The growth and characterization of the so obtained nanostructures has attracted increasing interest in the last few years due to their fascinating potential applications in integrated microelectronics. Apart from optical and electrical characterization, the structural analysis of these structures is usually performed by Scanning Electron Microscopy, Transmission Electron Microscopy or Atomic Force Microscopy. However, none of these techniques is suitable to exactly measure the total amount of deposited materials which is, together with the statistical distribution of the dimensions of the nanostructures, the most important information to be obtained. We have developed a new experimental set up for RBS analysis which, in connection with a computer simulation code, improves the thickness resolution of the technique of nearly one order of magnitude. With this experimental arrangement InAs/GaAs, InP/GaAs and InGaAs/GaAs nanostructures, nominally few monolayers (ML) thick can be analyzed by obtaining the total amount of deposited material, the fraction of the surface covered by the nanostructures and their maximum thickness.
\end{abstract}

\section{Introduction}

Rutherford Backscattering Spectrometry (RBS) is one of the most widely used methods to evaluate the total amounts of elements present in a sample, their stoichiometric ratio and, in many cases, their depth profile. This technique is usually employed to analyze continuous thin films. However, it has recently been shown [1,2] that RBS is also suitable to study isolated microstructures grown over a substrate of lighter mass.

In references [1,2] the MOVPE growth of InP on a (001) substrate was studied. It is now well established that in the case of the epitaxy of a film with a high lattice mismatch with respect to the substrate the growth proceeds via island formation, the islands being the peculiar sites of strain relaxation. We showed that in the case of InP islands, several tens of nanometers high, RBS analysis, in connection with a suitable computer simulation code, can give the maximum island height and the fraction of the surface covered by the growing islands, together with the total amount of deposited In.

$\left({ }^{*}\right)$ also Istituto di Ingegneria Gestionale dell'Università di Padova, Sede distaccata di Vicenza. 
This procedure, at least in principle, can be used to study self organized nanostructures which constitute one of the most challenging fields in semiconductor physics both from the fundamental point of view and for the potential application in micro and optoelectronics.

While semiconductor structures showing quantum confinement in 1 dimension (quantum wells) are now widely used in optoelectronics, structures exhibiting quantum confinement in 2 (quantum wires) or in 3 (quantum dots) dimensions are still under study to define the best technological route for their production.

In particular quantum dots should be very small in size, with base areas of less than $\sim 10^{-3} \mu \mathrm{m}^{2}$ and a few nanometer height. Moreover they should have a uniform distribution over the substrate with a high filling factor.

Many ways of producing nanostructures have been attempted including growth on vicinal surfaces [3], patterned substrates [4] and various ways of self organization of the islands [5,6]. The characterization probes include optical techniques as Low Temperature Photoluminescence and Raman Spectroscopy, and imaging techniques as Scanning Electron Microscopy (SEM), Transmission Electron Microscopy (TEM) and Atomic Force Microscopy (AFM).

As far as In compounds on GaAs substrates are under study, RBS is a suitable technique to measure the total amount of deposited material. However, when the island dimensions are such that the energy lost by the analyzing beam in crossing them is lower than the energy resolution of the detector, it is not possible to obtain supplementary information, mainly the depth distribution of the elements into the target which is strictly connected to the island height. If this limitation can be overcome the combined use of RBS, which intrinsically averages its information over an area of about $1 \mathrm{~mm}^{2}$ with other more focused topological techniques can give a detailed structural characterization of the nanostructures.

In this paper we demonstrate that by using an enhanced depth resolution RBS technique and by a careful computer simulation of the experimental spectra it is possible to characterize islands a few nanometers thick and covering only a small fraction of the substrate.

\section{Experimental}

InP/GaAs structures, nominally a few tens of nanometers thick, were grown at the ICTIMA-CNR Institute (Padova-Italy) with a low pressure MOVPE apparatus, whereas MBE growth was used at the MASPEC-CNR Institute (Parma-Italy) in order to obtain InAs/GaAs structures nominally $3 \mathrm{ML}$ thick. In this case the substrate temperature ranged from $440{ }^{\circ} \mathrm{C}$ to $530{ }^{\circ} \mathrm{C}$. Further details on the growth procedures will be given elsewhere.

The RBS analyses were performed at the AN2000 and CN accelerators at the Laboratori Nazionali di Legnaro (Padova-Italy). A $2 \mathrm{MeV} \mathrm{He}^{+}$beam was used. A Si surface barrier detector having $11 \mathrm{keV}$ energy resolution (FWHM) was located under the incident beam at $170^{\circ}$.

To improve the depth resolution we operated both on: i) the apparent sample thickness as seen by the incident beam, and ii) the experimental set up in order to minimize the kinematic energy spread due to the acceptance angle of the detector and to the dimension of the beam at the sample surface.

i) The apparent sample thickness has been increased by a factor $1 / \cos \theta$ by rotating the sample of an angle $\theta$ around a vertical axis. The best operating conditions were found for $80^{\circ} \leq \theta \leq 83^{\circ}$ giving a magnification factor $F$ of the apparent depth: $5.76 \leq F \leq 8.21$.

ii) When the sample is rotated of an angle $\theta$ the beam spot on the sample surface is also enlarged of a factor $F$ in the horizontal direction. A rectangular diaphragm, $1 \mathrm{~mm}$ high and $0.25 \mathrm{~mm}$ wide was then placed at the scattering chamber entrance so that, at the maximum sample tilt, the beam spot size was $2 \times 1 \mathrm{~mm}^{2}$. The sample-detector distance was $70 \mathrm{~mm}$. A vertical collimator, placed on the detector surface reduced the scattering angle spread to $\pm 0.4^{\circ}$. 


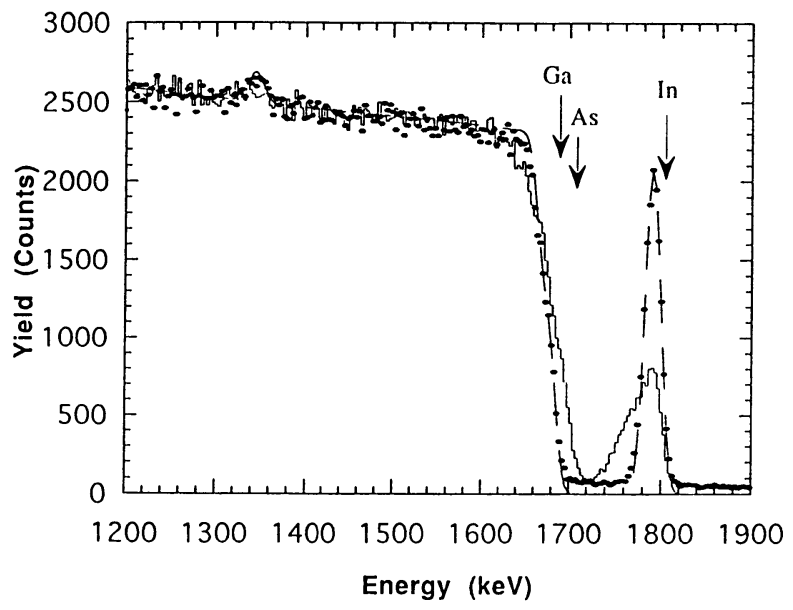

Fig. 1. - RBS spectra of a thin continuous InP/GaAs film (full dots-broken line) and of InP islands, MOVPE grown on GaAs. The arrows mark the scattering energies from the various elements at the surface. $\left(2 \mathrm{MeV} \mathrm{He}^{+}, \theta_{\text {scatt }}=170^{\circ}\right)$.

A careful energy calibration was performed with the aid of several monoelemental targets giving an "energy per channel" $=3.100 \pm 0.013 \mathrm{keV}$. The absolute In amount was obtained by calibrating the spectra against standard samples having a precision better than $1.5 \%$ [7].

A computer code developed at Padova [8] was used to simulate the experimental spectra. The simulation procedure is the following: a trial concentration profile as a function of depth is assumed for each of the elements present in the target. Then, by taking into account these profiles the experimental spectrum is simulated by computing the yield and the energy loss and straggling along the in- and out-going path for successive layers which can be as thin as $10^{15} \mathrm{at} / \mathrm{cm}^{2}$, whereas no limitation is given on the minimum step thickness of the concentration profiles. The simulated yield is then convoluted with the experimental detector resolution and normalized for the experimental solid angle and integrated beam charge. The concentration profiles are changed until a reasonable fit to the experimental spectrum is obtained within the statistics.

Finally RBS results were compared to AFM topographies performed with a Discoverer Topometrix at the LAMEL-CNR Institute in Bologna,Italy.

\section{Results}

The general rule for RBS analysis is that, provided the total energy loss is at least twice the energy resolution, the energy width of the signal gives the film thickness while the signal height gives the stoichiometric ratio or the surface coverage fraction for discontinuous films.

Figure 1 displays the superimposition of two experimental spectra from a thin continuous InP/ GaAs layer and from a sample in which InP islands, several tens of nanometers thick partially cover the substrate. The analysis of this sample can be performed as follows: the ratio of its In yield at the surface to that of the continuous film gives the surface coverage fraction of the islands whereas the low-energy tail of the In signal gives the measure of the sample "roughness" $[1,2]$. From the In depth profile the surface coverage fraction and the maximum island thickness can thus be derived. 
This analysis procedure can apply only if the island thickness is large enough. In fact with a $11 \mathrm{keV}$ energy resolution detector the depth resolution achievable when no tilt angle is applied to the target is about $18 \mathrm{~nm}$ (FWHM), a value which is too high to perform any depth analysis on very thin samples, like the present InAs samples.

For InAs, even under extreme grazing incidence and emergence $\left(83^{\circ}\right)$ the total energy loss reaches twice the energy resolution for thicknesses of the order of $4 \mathrm{~nm}(14 \mathrm{ML})$. This means that this condition could be met in our samples (3 ML equivalent deposition) only for surface coverage lower than $20 \%$. For higher coverage, and/or thinner islands, the standard height and width analysis cannot be employed and we decided to concentrate our analysis on the "shape" of the In peak as a function of different InAs distributions on the surface with the constraint of fixed In total amount.

In fact, by taking constant the absolute amount of In, slight differences in its depth distribution strongly affect the shape of the RBS signal and, by a careful comparison of the simulated spectrum to the experimental one the main features of the islands size and distribution can be derived.

Figure 2 show's the computer simulated spectra for a $3 \times 10^{15} \mathrm{InAs} / \mathrm{cm}^{2}$ analyzed with a $83^{\circ}$ tilt angle. The simulations are performed: i) by assuming that the surface is covered by a continuous film $1.5 \mathrm{~nm}$ thick, ii) by supposing the formation of islands (with box shape for simplicity) covering $50 \%$ of the substrate up to a thickness of $6 \times 10^{15} \mathrm{InAs} / \mathrm{cm}^{2}(3 \mathrm{~nm})$ and iii) by considering a continuous film, $2 \mathrm{ML}$ thick, and the remaining In in islands covering $30 \%$ of the surface with a linear distribution of thicknesses up to a thickness of $1.24 \times 10^{16} \mathrm{InAs} / \mathrm{cm}^{2}$ (about $6.5 \mathrm{~nm}$ ). Only small differences can be observed in the GaAs part of the spectrum. The In signals (expanded by a factor of 3 in the figure) do not differ in their width at half maximum, as it is expected, since all the three supposed thicknesses do not imply an energy loss greater than twice the detector resolution. However from the comparison of the three simulated spectra some conclusions can be drawn. First, if a continuous InAs layer is assumed to cover the substrate, the maximum of the In signal appears at the right energy for scattering at the surface ( $\mathrm{i}$ and iii) whereas if a partial coverage of the substrate is assumed (ii) the signal is shifted towards lower energies. Moreover, even if the FWHM of the three simulated spectra do not appreciably differ, the low energy side of the spectra is progressively shifted towards lower energies by increasing the maximum depth of the simulation profiles. As a consequence the most significant features of the In experimental spectrum are the energy position of the peak maximum, which is related to the presence of a continuous film and the low energy tail of the signal, which is related to the island maximum thickness.

This RBS analysis technique was applied to the InAs/GaAs nanostructures.

Preliminary AFM measurements on the samples had shown that the shape of the islands was globular with much higher lateral dimensions than height. In this situation the assumption of considering the islands as small discs is, as a first approximation, justified.

The total amount of In in the samples was determined to be $(2.12 \pm 0.1) \times 10^{15} \mathrm{In} / \mathrm{cm}^{2}$ for all the samples, indicating an In content $20 \%$ higher than the nominal one.

Figure 3 shows the In part of the RBS spectra for two samples, respectively grown at $T=440^{\circ} \mathrm{C}$ and $T=530^{\circ} \mathrm{C}$. Although the In peak position and total dose in the samples are the same, differences in the shape of the signals are evident: for the sample grown at higher temperature both a lower signal height and a wider low energy tail suggest a lower surface coverage with a corresponding higher maximum height of the islands.

The energy position of the In peaks suggests the presence of a continuous film underlying the islands, this is in agreement with other literature results $[9,10]$ which indicate that the InAs/GaAs system grows in the Stransky-Krastanov mode with a $1.7 \mathrm{ML}$ thick film wetting the substrate. We tried to simulate the spectra by assuming the presence of a 1,2 or 3 ML thick continuous film (the integer ML steps were chosen for computational ease) and we found that for all the samples 


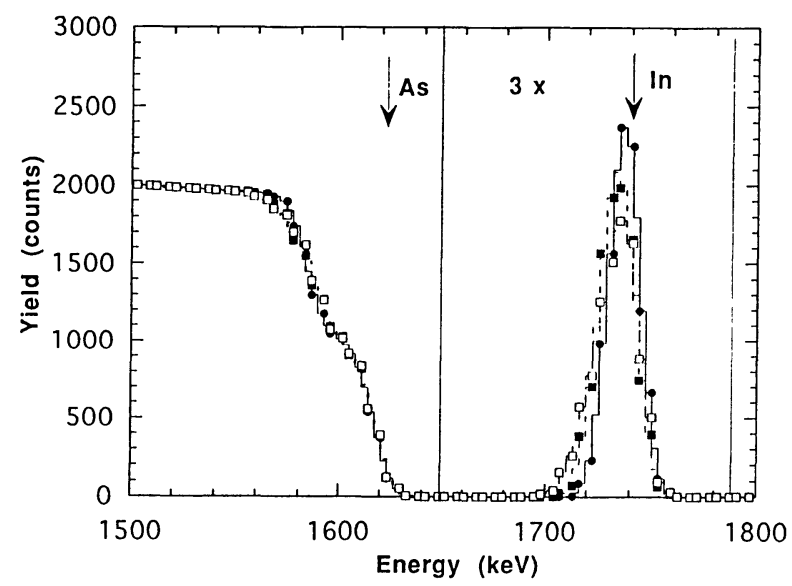

Fig. 2. - Computer simulated RBS spectra for $3 \times 10^{15} \mathrm{InAs} / \mathrm{cm}^{2}$ structures with different In distribution profiles: full dots- continuous layer $(1.5 \mathrm{~nm}$ thick), full squares- islands covering $50 \%$ of the substrate $(3 \mathrm{~nm}$ thick), open squares- 2 ML thick continuous film, plus islands covering $30 \%$ of the surface with heights up to $6.5 \mathrm{~nm} .\left(2 \mathrm{MeV} \mathrm{He}^{+}, \theta_{\text {scatt }}=170^{\circ}, \theta_{\text {tilt }}=83^{\circ}\right)$.

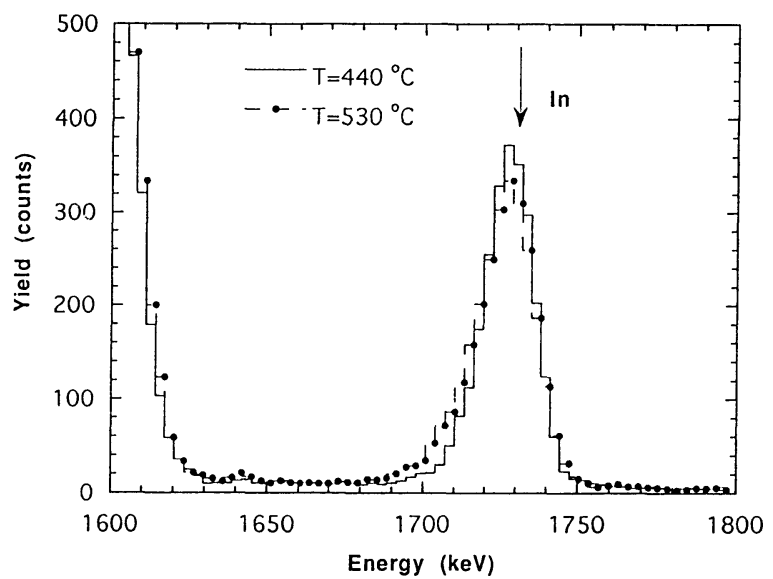

Fig. 3. - RBS experimental spectra of two InAs/GaAs structures, 3 ML nominal deposition: full dots$T_{\text {growth }}=530^{\circ} \mathrm{C}$, continuous line- $T_{\text {growth }}=440^{\circ} \mathrm{C}$. Only the In part of the spectra is displayed. $\left(2 \mathrm{MeV} \mathrm{He}^{+}\right.$, $\left.\theta_{\text {scatt }}=170^{\circ}, \theta_{\text {tilt }}=80^{\circ}\right)$.

a $2 \mathrm{ML}$ film at the interface best simulates the trailing edge of the spectra. This implies that for these samples less than $50 \%$ of the deposited material has gone into island formation. Particular care was devoted in defining the uncertainty in the maximum island height determination, given by the leading edge of the spectra, and we found that this uncertainty (for a $83^{\circ}$ tilt) is equal to $\pm 2 \times 10^{15} \mathrm{InAs} / \mathrm{cm}^{2}$, corresponding to a thickness of $\pm 1 \mathrm{~nm}$. Once the thickness of the continuous film has been determined together with the maximum island height, the surface coverage fraction is univocally determined from the constraint of the conservation of the total dose. By varying the In dose within its error bar the surface coverage fraction varies of \pm 0.05 . 


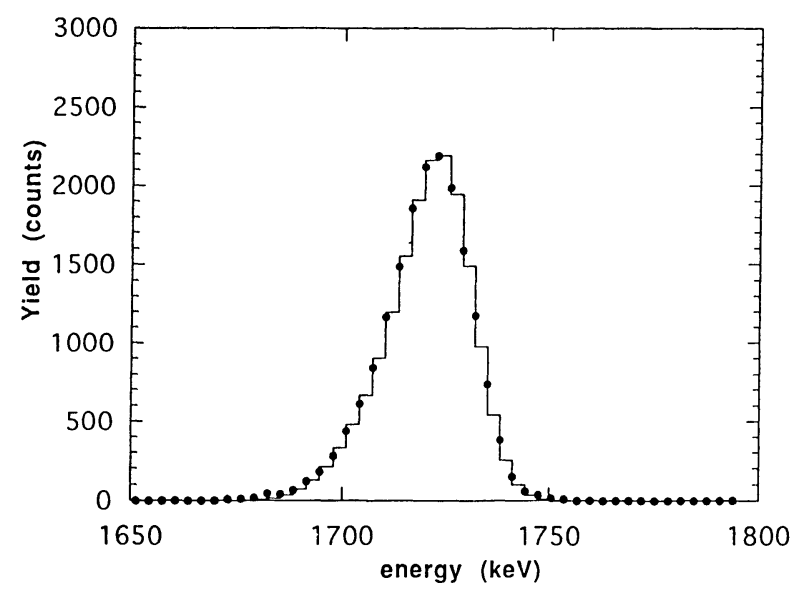

Fig. 4. - Indium RBS signal for a InAs sample grown at $T=440^{\circ} \mathrm{C}$ (full dots) together with its best simulated spectrum (continuous line). Details on the simulation profile are given in the text. $\left(2 \mathrm{MeV} \mathrm{He}^{+}\right.$, $\theta_{\text {scatt }}=170^{\circ}, \theta_{\text {tilt }}=83^{\circ}$ ).

Figure 4 shows the In part of the experimental spectrum for the $440^{\circ} \mathrm{C}$ grown InAs sample (full dots) together with its best simulation. The concentration profile used to simulate the spectrum consists of $2 \mathrm{ML}$ of InAs on top of which islands, $6 \mathrm{~nm}$ thick, are grown, covering $16 \%$ of the surface.

A comparison of the present results with those obtained by AFM indicates a rather good agreement even though the discrepancies are somewhat higher than the estimated error bar. Details on the analyses will be presented elsewhere.

The same procedure has been applied to the analysis of other samples. Preliminary results indicate that the island height lowers with temperature, consequently the filling factor increases.

\section{Conclusions}

This is the first time that RBS technique is used in depth enhancing geometry in order to study non planar films. The main outcome of the analysis is the possibility of discriminating about the presence of a thin continuous film which covers the substrate below the islands. The presence of this film, whose thickness in the case of the system studied in this work, can be determined with a maximum uncertainty of $\pm 0.5 \mathrm{ML}$, definitely indicates that the island growth proceeds in the Stransky-Krastanov mode. The island height can be determined with a precision of $\pm 1 \mathrm{~nm}$ and together with the measure of the total amount of deposited material allows, in principle, to give the surface coverage with an error of \pm 0.05 . However this limit in the surface coverage determination can be reached only if the actual shape of the islands is considered in the simulation procedure. If not, and this is the case of the present work, the error can be considerably higher.

We believe that the combined use of this new experimental approach which averages its information over large areas with other more focused complementary techniques like AFM, SEM and TEM will allow a deeper insight in the nanostructures analysis. 


\section{Acknowledgements}

The authors acknowledge S. Franchi and A. Bosacchi who performed the MBE growth of InAs samples and G. Rossetto and A. Camporese who performed the MOVPE growth of InP/GaAs samples. We are also grateful to A. Sambo for his skillful technical assistance.

\section{References}

[1] Romanato F., Berti M., Mazzer M., Drigo A.V., Lazzarini L., Franzosi P., Salviati G.C. and Bertone D., Mikrochim. Acta 114/115 (1994) 431.

[2] Berti M., Drigo A.V., Mazzer M., Romanato F., Lazzarini L., Franzosi P., Salviati G.C. and Bertone D., Mater. Sci. Eng. B28 (1994) 214.

[3] Nötzel R., Temmyo J., Kamada H., Furuta T. and Tamamura T., Appl. Phys. Lett. 65 (1994) 457.

[4] Arakawa Y., Nagamune Y., Nishioka M. and Tsukamoto S., Semicond. Sci. Technol. 8 (1993) 1082.

[5] Ahopelto J., Atsushi Yamaguchi A., Nishi K., Usui A. and Sakaki H., Jpn. J. Appl. Phys. 32 (1993) L32.

[6] Marzin J.Y., Gérard J.M., Izrael A., Barrier D. and Bastard G., Phys. Rev. Lett. 73 (1994) 716.

[7] Cohen C., Davies J.A., Drigo A.V. and Jackman T.E., Nucl. Instr. Meth. 218 (1983) 147.

[8] Berti M., Carnera A., unpublished.

[9] Goldstein L., Glas F., Marzin J.Y., Charasse M.N. and Le Roux G., Appl. Phys. Lett. 47 (1985) 1099.

[10] Gérard J.M., Appl. Phys. Lett. 61 (1992) 2096. 